
\title{
$-$
}

\section{Autophagy Protects Against Senescence and Apoptosis via the RAS-Mitochondria in High-Glucose-Induced Endothelial Cells}

\author{
Fei Chen ${ }^{\mathrm{a}}$ Bin Chen ${ }^{\mathrm{a}}$ Fen-Qiang Xiao ${ }^{\mathrm{a}}$ Yu-Tao Wu $\mathrm{Wu}^{\mathrm{a}}$ Ri-Hong Wang ${ }^{\mathrm{a}}$ Ze-Wei Sun ${ }^{\mathrm{a}}$ \\ Guo-Sheng Fu ${ }^{b}$ Yun Mou ${ }^{c}$ Wu Tao ${ }^{a}$ Xiao-Sheng Hua Shen-Jiang Hu${ }^{a}$ \\ Institute of Cardiology, The First Affiliated Hospital, College of Medicine, Zhejiang University, \\ 'Department of Cardiology, Sir Run Run Hospital, College of Medicine, Zhejiang University, \\ 'Department of Ultrasound, The Affiliated Hospital, College of Medicine, Zhejiang University, \\ Hangzhou, PR China
}

\section{Key Words}

Autophagy $\cdot$ Senescence $\cdot$ Apoptosis $\cdot$ High glucose $・$ Endothelium $・$ Rennin-angiotensin

\begin{abstract}
Backgrounds: Autophagy is an important process in the pathogenesis of diabetes and plays a critical role in maintaining cellular homeostasis. However, the autophagic response and its mechanism in diabetic vascular endothelium remain unclear. Methods and Results: We studied high-glucose-induced renin-angiotensin system (RAS)-mitochondrial damage and its effect on endothelial cells. With regard to therapeutics, we investigated the beneficial effect of angiotensin-converting enzyme inhibitors (ACEIs) or angiotensin II type 1 receptor blockers (ARBs) against high-glucose-induced endothelial responses. High glucose activated RAS, enhanced mitochondrial damage and increased senescence, apoptosis and autophagicresponses in endothelial cells, and these effects were mimicked by using angiotensin II (Ang). The use of an ACEI or ARB, however, inhibited the negative effects of high glucose. Direct mitochondrial injury caused by carbonyl cyanide 3-chlorophenylhydrazone (CCCP) resulted in similar negative effects of high glucose or Ang and abrogated the protective effects of an ACEI or ARB. Additionally, by impairing autophagy, high-glucose-induced senescence and apoptosis were accelerated and the ACEI- or ARB-mediated beneficial effects were abolished. Furthermore, increases in FragEL ${ }^{\text {TM }}$ DNA Fragmentation (TUNEL)-positive cells, $\beta$-galactosidase activation and the expression of autophagic biomarkers were revealed in diabetic patients and rats, and the treatment with an ACEI or ARB decreased these responses. Conclusions: These data suggest that autophagy protects against senescence and apoptosis via RAS-mitochondria in high-glucose-induced endothelial cells.
\end{abstract}

Copyright $@ 2014$ S. Karger AG, Basel 


\section{Introduction}

The vascular endothelium serves as a key player in the pathogenesis of diabetic complications via abnormal vascular tension, augmented vascular inflammation and increased apoptotic endothelial cells $[1,2]$. The occurrence of endothelial autophagy, apoptosis and senescence has been described in diabetic rats or high-glucose-treated endothelial cells [3-5]. Autophagy is known a "housekeeper" function for maintaining cellular homeostasis through degradation and the recycling of damaged organelles and misfolded proteins [6]. Apoptosis, defined as programmed cell death, also serves a homeostasis function through the regulation of cell death upon stress [7]. Senescence also contributes to the development of diabetic complications and age-related diseases [8]. There are complex relationships within autophagy, apoptosis and senescence. Excited or abrogated autophagy may enhance apoptosis, and a reduction in autophagy may accelerate or delay senescence. Additionally, senescence appears to be resistant to apoptosis, and apoptosis suppresses autophagy [9-11]. Autophagy, apoptosis and senescence may be initiated by similar stimuli, such as reactive oxygen species (ROS), mitochondrial damage and cytoplasmic $\mathrm{Ca}^{2+}[3,4]$. Understanding the relationships among autophagy, apoptosis and senescence and their mechanisms are hot topics in current scientific researches.

The renin-angiotensin system (RAS) plays a pivotal role in cardiac development and the pathogenesis of cardiovascular disease, including cellular hypertrophy, proliferation and apoptosis [12-14]. Disrupting RAS has been shown to provide significant benefit for cellular function, and this effect has been shown to be reliant on mitochondrial-ROS [12-15]. Angiotensin II (Ang) exposure or overexpression of Ang type 1 receptor (AGTR1)-coupledG-protein, Gaq, induces excessive ROS production and triggers autophagy, senescence and apoptosis in cardiomyocytes and smooth muscle cells $[8,16,17]$. However, whether RAS activity is increased and whether there is benefit to the use of angiotensin-converting enzyme inhibitors (ACEIs) or angiotensin II type 1 receptor blockers (ARBs) in diabetic patients without cardiovascular complications remains controversial. Moreover, whether RAS induces autophagy, apoptosis and senescence through mitochondrial damage and mitochondrial-ROS in high-glucose-treated human umbilical vein endothelial cell (HUVECs) remains poorly understood. The aim of this study was to test whether high-glucose-induced RAS activation and increased RAS activity cause imbalances in autophagy, senescence and apoptosis in endothelial cells and whether autophagy may play an important role in these mechanisms.

\section{Materials and Methods}

All animal testing procedures were approved by the Ethics Committee for the Use of Experimental Animals at Zhejiang University and conformed with the "Guidelines for the Care and Use of Laboratory Animals" published by National Academy Press (National Institutes of Health Publication No. 85-23, revised 1996). The rats were anesthetized using $2 \%$ pentobarbital $(\mathrm{v} / \mathrm{v} ; 0.3 \mathrm{ml} / 100 \mathrm{~g}, \mathrm{v} / \mathrm{w})$ by i.p. injection. The human study conforms with the principles outlined in the Declaration of Helsinki.

Reagents

MAP1LC3B and BECN1 siRNA were purchased from GenePharma (Shanghai, China). The chemical reagents (3-MA, Compound C (CC), 5-aminoimidazole-4-carboxyamide ribonucleoside (AICAR)), and the TUNEL-kit were purchased from Merck (Darmstadt, Germany). Losartan was obtained from Cayman (Ann Arbor, USA), and Benazepril was a kind gift from Novartis (Basel, Switzerland). The anti-phospho-ULK, anti-AMPK, anti-p62, anti-LC3, anti-cytC, anti-ACE, anti-AGTR1, anti- $\beta$-actin and secondary antibodies were obtained from Epitomics (Burlingame, USA); the anti-phospho-AMPK, anti-p21, anti-beclin1, anti-bcl2 and anti-bax antibodies were purchased from Cell Signaling (Denver, USA); the anti-ULK antibody was obtained from Abcam (HK, China). MitoTracker Red and lipofectamine RNAiMax were purchased from Invitrogen (Carlsbad, USA), and Fluo-4AM was obtained from Dojindo (Kumamoto, Japan). The cell cycle-kit and the cell apoptosis-kit were purchased from BD (Franklin Lakes, USA). JC-1, ROS, SA- $\beta$-gal-kit and protein lysis 
buffer were obtained from Beyotime (Haimen, China). The NADPH-kit was obtained from AAT (Sunnyvale, USA). Other unmentioned reagents were purchased from Sigma (St. Louis, USA).

\section{Cell culture}

The human umbilical vein endothelial cell (HUVEC) line and endothelial cell media (ECM) were purchased from ScienCell (Carlsbad, California, USA). The HUVECs were grown in ECM (glucose: $5.5 \mathrm{mM}$ ). The cells were starved by growing without FBS for $12 \mathrm{~h}$ and were then exposed to
Table 1. Oligonucleotide sequences of the primers and siRNAs

\begin{tabular}{|c|c|c|}
\hline Genes & Primers & Protocol \\
\hline \multirow[t]{2}{*}{ ACE 1} & Forward: 5'-CCTGTTGGATATGGAAACCACCTAC-3' & \\
\hline & Reverse: 5'-TCCCATGCCCATAACAGGTC-3' & \\
\hline \multirow[t]{2}{*}{ AT1 } & Forward: 5'-TCCAGATTGTTCTGTCCAGTTTCC-3' & stage $1: 95^{\circ} \mathrm{C}, 30 \mathrm{sec}$ \\
\hline & Reverse: 5'-TCTGCAACTTGACGACTACTGCTTA-3' & stage $2: 95^{\circ} \mathrm{C}, 5 \mathrm{sec}$ \\
\hline \multirow[t]{2}{*}{ AT2 } & Forward: 5'-GGCACTAAGCAAGCTGATTTATGA-3' & $55^{\circ} \mathrm{C}, 30 \mathrm{sec}$ \\
\hline & Reverse: 5'-CGAAGTGAAGACCGCTGGTA-3' & $72^{\circ} \mathrm{C}, 35 \mathrm{sec}$ \\
\hline \multirow[t]{2}{*}{ GAPDH } & Forward: 5'-AAGGTGAAGGTCGGAGTCA-3' & 40 cycles \\
\hline & Reverse: 5'-GGAAGATGGTGATGGGATTT-3' & \\
\hline \multirow[t]{2}{*}{ NC } & Sense: 5'-UUCUCCGAACGUGUCACGUTT-3' & \\
\hline & Anti-sense: 5'-ACGUGACACGUUCGGAGAATT-3' & \\
\hline \multirow[t]{2}{*}{ GAPDH } & Sense: 5'-GUAUGACAACAGCCUCAAGTT-3' & \\
\hline & Anti-sense: 5'-CUUGAGGCUGUUGUCAUACTT-3' & \\
\hline \multirow[t]{2}{*}{ BECN1 } & Sense: 5'-GCUGCCGUUAUACUGUUCUTT-3' & \\
\hline & Anti-sense: 5'-AGAACAGUAUAACGGCAGCTT-3' & \\
\hline \multirow[t]{2}{*}{ MAP1LC3B } & Sense: 5'-GCCCUCUACUGAUUGUUAATT-3' & \\
\hline & Anti-sense: 5'-UUAACAAUCAGUAGAGGGCTT-3' & \\
\hline
\end{tabular}
glucose at concentrations of $5.5,11,16.5,22,27.5$ or $33 \mathrm{mM}$ for $72 \mathrm{~h}$. An equal concentration (27.5 mM) of mannitol was added to eliminate the effect of osmotic pressure caused by high glucose. At $6 \mathrm{~h}$ before the cells were treated with benazepril $(100 \mu \mathrm{M})$, losartan $(50 \mu \mathrm{M})$ or PD123319 $(10 \mu \mathrm{M})$, the cells were cultured in the presence of glucose $(33 \mathrm{mM})$ for an additional $72 \mathrm{~h}$ to evaluate the role of RAS. Ang $(1 \mu \mathrm{M})$ was appended for $72 \mathrm{~h}$ to active RAS. To impair autophagy, chloroquine $(10 \mu \mathrm{M})$ for $6 \mathrm{~h}$ or 3 -MA $(5 \mathrm{mM})$ for $24 \mathrm{~h}$ was appended before glucose administration. Additionally, CC $(1 \mu \mathrm{M})$ or AICAR $(0.5 \mathrm{M})$ was appended for $24 \mathrm{~h}$ before glucose administration to regulate AMPK phosphorylation.

Real time reverse transcription PCR (RT-PCR)

Total RNA was converted to cDNA using murine leukemia virus reverse transcriptase (Promega, Mannheim, Germany). The RT-PCR was executed using an ABI 7500 system using SYBR Premix chemistry (Takara, Shiga, Japan). The primers (Takara, Shiga, Japan) are displayed in Table1.

Measurement of ROS, $\triangle \Psi$ m or cytoplasmic $\mathrm{Ca}^{2+}$

After staining with DCHF-DA (ROS) for $30 \mathrm{~min}$, JC-1 $(0.3 \mu \mathrm{g} / \mathrm{ml}, \Delta \Psi \mathrm{m})$ for $30 \mathrm{~min}$ or Fluo4-AM (1 $\mu \mathrm{M}$, cytoplasmic $\mathrm{Ca}^{2+}$ ) for $45 \mathrm{~min}$, respectively, cells were examined using fluorescence microscopy or flow cytometry.

Cell cycle analysis

Cells were fixed using 70\% ethanol overnight, then incubated with $50 \mu \mathrm{g} / \mathrm{ml}$ propidium iodide (PI), $100 \mu \mathrm{g} / \mathrm{ml}$ DNAase-free RNAase and 2\%o Triton-X-100 for $45 \mathrm{~min}$, and then analyzed using flow cytometry.

Cell apoptosis analysis

Cells were incubated with $5 \mu \mathrm{l}$ ANNEXIN-V-FITC and $2.5 \mu \mathrm{g} / \mathrm{ml}$ PI for $15 \mathrm{~min}$ prior to flow cytometry analysis.

\section{$S A-\beta$-gal detection}

Cells were processed using the SA- $\beta$-gal-kit according to the manufacturer's instructions. Briefly, the cells were fixed for $15 \mathrm{~min}$, then incubated with 5-bromo-4-chloro-3-inolyl-b-D-galactoside ( $\beta$-gal) substrate for $24 \mathrm{~h}$ at $37^{\circ} \mathrm{C}$ and then imaged.

Transmission electron microscopy

After the $72 \mathrm{~h}$ treatment, the cells were harvested and fixed in 3\% glutaraldehyde and then dehydrated in a graded series of ethanol solutions. After processing, the samples were observed using a transmission electron microscope.

\section{Measurement of $N A D P H$}

Cells were lysed in ice for $15 \mathrm{~min}$ and centrifuged. Supernatant or NADPH standard diluents were mixed with the reaction solution. The mixtures were incubated for $1 \mathrm{~h}$ and then measured for absorbance at $570 \mathrm{nM}$. 
Chen et al.: RAS and Autophagy, Senescence, Apoptosis

\section{SAHF detection}

Cells were fixed using $90 \%$ ice-cold methanol for $10 \mathrm{~min}$ and then treated with $0.1 \mu \mathrm{g} / \mathrm{ml}$ anti-fade DAPI for 2 min. The samples were analyzed using a fluorescence microscope.

\section{TUNEL detection}

Tissue sections were deparaffinized and hydrated and then tissue slices were permeabilized using protease $\mathrm{K}$ and incubated with terminal deoxynucleotidyl transferase (TdT) enzyme for 90 min. DAB was applied for $30 \mathrm{sec}$. The samples were imaged using a microscope.

\section{siRNA transfection}

Cells were transfected with either control RNA, siRNA targeted human LC3 and Beclin1 using the lipofectamine RNAiMax procedure. The siRNA sequences are shown in Table1.

\section{Fluorescence imaging of LC3 or cytC release}

After staining with $200 \mathrm{nM}$ MitoTracker Red for $30 \mathrm{~min}$ (For cytC release), cells were fixed, permeabilized and blocked. Cells were incubated sequentially with anti-LC3 or anti-cytC solution at $4{ }^{\circ} \mathrm{C}$ overnight and a Dy-Light ${ }^{\mathrm{TM}}$ 488-conjugated secondary antibody solution at room temperature for $1 \mathrm{~h}$, and then $1 \mu \mathrm{g} / \mathrm{ml}$ anti-fade DAPI solution for $2 \mathrm{~min}$. Cells were analyzed using a fluorescence microscope.

\section{Western blotting}

Cells were lysed in ice for $45 \mathrm{~min}$. Samples of equal concentrations of protein were subjected to 8-12\% SDS-PAGE, transferred to PVDF membranes and blocked using 5\% non-fat milk. The membranes were coated with primary antibodies overnight and then incubated with HRP-conjugated secondary antibodies for $1 \mathrm{~h}$. Immunoblots were developed using enhanced chemiluminescence reagents (Bio-Max, Israel).

\section{Immunohistochemical analysis}

After deparaffinizing, tissues were hydrated and blocked and then incubated sequentially with primary antibodies for $2 \mathrm{~h}$ and then antidigoxigenin-conjugated secondary antibodies for $1 \mathrm{~h}$ (tissue slices were washed carefully between antibody solutions). After incubating with DAB, the samples were imaged.

\section{Animal model}

Blood glucose levels were measured at $72 \mathrm{~h}$ after STZ (70 mg/kg) i.p. injections into SD rats (180-220 g). Rats with glucose levels $>16.4 \mathrm{mM}$ were considered to have undergone successful induction of diabetes. The diabetic rats were randomly divided into three groups and administered intragastric benazepril (10 $\mathrm{mg} / \mathrm{kg} / \mathrm{d})$, losartan $(80 \mathrm{mg} / \mathrm{kg} / \mathrm{d})$ or equal volume of physiological saline for 8 weeks.

\section{Bioassay of vasoreactivity}

After the rats were anesthetized, a section of the thoracic aorta was rapidly dissected out and immersed in chilled Krebs solution ( (mM) NaCl, 118; $\mathrm{KCl}, 4.7 ; \mathrm{MgSO}_{4} \cdot 7 \mathrm{H}_{2} \mathrm{O}, 1.2 ; \mathrm{KH}_{2} \mathrm{PO}_{4}, 1.2 ; \mathrm{CaCl}_{2}, 2.5 ; \mathrm{NaHCO}_{3}, 25$; glucose, 11 ; and bubbled with $95 \% \mathrm{O}_{2}+5 \% \mathrm{CO}_{2}(\mathrm{pH} 7.4)$ ). The aortic rings were cut into 3-5-mm lengths after carefully removing the perivascular tissue. After equilibrating at a $2.0 \mathrm{~g}$ initial load for $60 \mathrm{~min}$, the rings were precontracted using phenylephrine $(1 \mu \mathrm{M})$. After the developed tension reached its maximum, the rings were relaxed using cumulative doses of acetylcholine (0.001-10 $\mu \mathrm{M})$ or SNP $(0.001-10 \mu \mathrm{M})$.

\section{Human Study}

Informed consent was obtained from all participants in accordance with the guidelines of the Human Subjects Committee of the Medical Ethical Commission of the First Affiliated Hospital of Zhejiang University (Zhejiang, China). All samples were obtained from the left gastric artery after subtotal gastrectomy. We selected the left gastric artery as a target because subtotal gastrectomy was the most popular operation in patients without tumors, and the left gastric artery was of sufficient size (5-8 cm in length) for endothelial cell collection. Patients with hypertension, heart disease or tumors were excluded. After carefully washing the samples, the arterial inner lumen was filled with $0.25 \%$ trypsin and digested for $10 \mathrm{~min}$ at $37^{\circ} \mathrm{C}$, and then the arterial inner lumen was swilled with PBS three times. The trypsin and PBS solution were collected and centrifuged. After removing the supernatants, the cell sediments were added to a lysis buffer and western blotting was completed. 
Fig. 1. Pharmacological induction of RAS by high glucose or Ang. A. HUVECs were treated with glucose at $5.5,11,16.5,22$ or $27.5 \mathrm{mM}$ for $72 \mathrm{~h}$ or $33 \mathrm{mM}$ for 72,48 , 24 or 6 h. mRNA levels and the protein levels of ACE1 and AGTR1 were determined. High glucose significantly increased ACE1 and AGTR1 mRNA and protein expression $\left(n=3,{ }^{*} p<0.05\right)$. Mannitol was used to exclude effects caused by increased osmotic pressure. Con, control; Man, mannitol. B. Cells were treated with benazepril $(100 \mu \mathrm{M})$, losar$\tan (50 \mu \mathrm{M})$ or PD123319 (10 $\mu \mathrm{M}$ ) and high glucose (33 mM) for 72 h. Benazepril and losartan significantly decreased high-glucosemediated ACE1 and AGTR1 mRNA and protein expression $(\mathrm{n}=3, \quad \# \mathrm{p}<0.05)$. C. HUVECs were treated with glucose (33 mM) or Ang (1 $\mu \mathrm{M}$ ) for $72 \mathrm{~h}$. Ang mimicked high-glucose-induced ACE1 and AGTR1 protein expression.

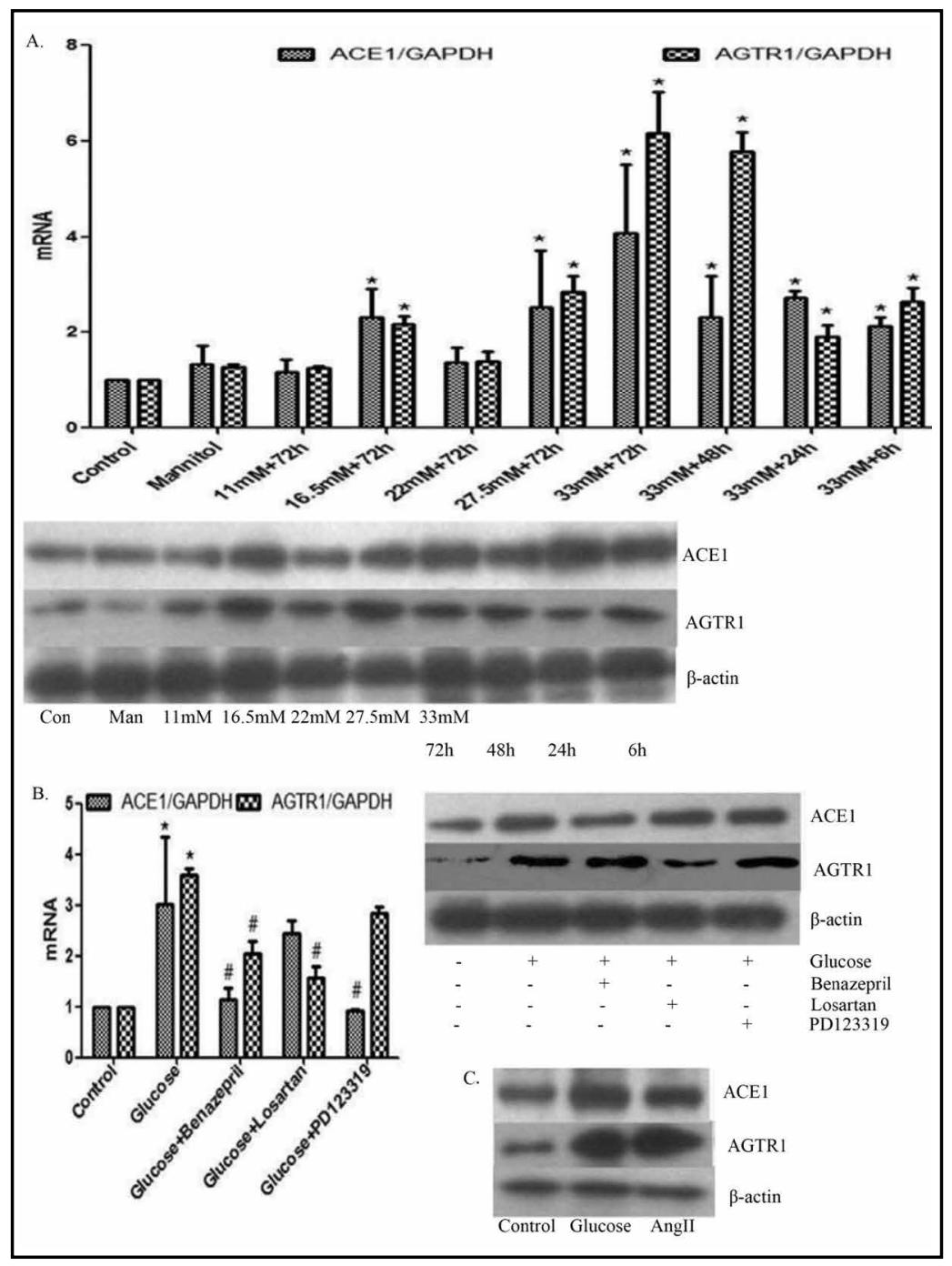

Statistical analyses

Significant differences were determined using SPSS14.0 software, and all data are presented as the mean \pm SEM. Comparisons were performed using one-way ANOVA followed by Tukey's HSD test. The data for vascular contraction and vasodilatation were analyzed using two-way ANOVA. A value of $\mathrm{p}<0.05$ was considered to be statistically significant.

\section{Results}

High glucose increased ACE1 and AGTR1 expression

To assess whether high-glucose induces RAS activation in endothelial cells, we firstly examined the expression of ACE1 and AGTR1 mRNA and protein. As shown in Fig. 1A, glucose induced ACE1 or AGTR1 mRNA and protein expression but not AGTR2 expression (data not shown). The expression of ACE1 or AGTR1 reached its maximum value at $33 \mathrm{mM}$ glucose for $72 \mathrm{~h}$, whereas incubation with mannitol did not change RAS expression.

RAS participates in regulating high-glucose-induced mitochondrial dysfunction in HUVECS

As shown in Fig. 2A, following a 72-h treatment with $33 \mathrm{mM}$ glucose, we observed a nearly $30 \%$ reduction in mitochondrial membrane potentials $(\Delta \Psi \mathrm{m})$. In parallel to the loss in $\Delta \Psi \mathrm{m}, \mathrm{cytC}$ was released into the cytoplasm. Furthermore, glucose treatment resulted in a 


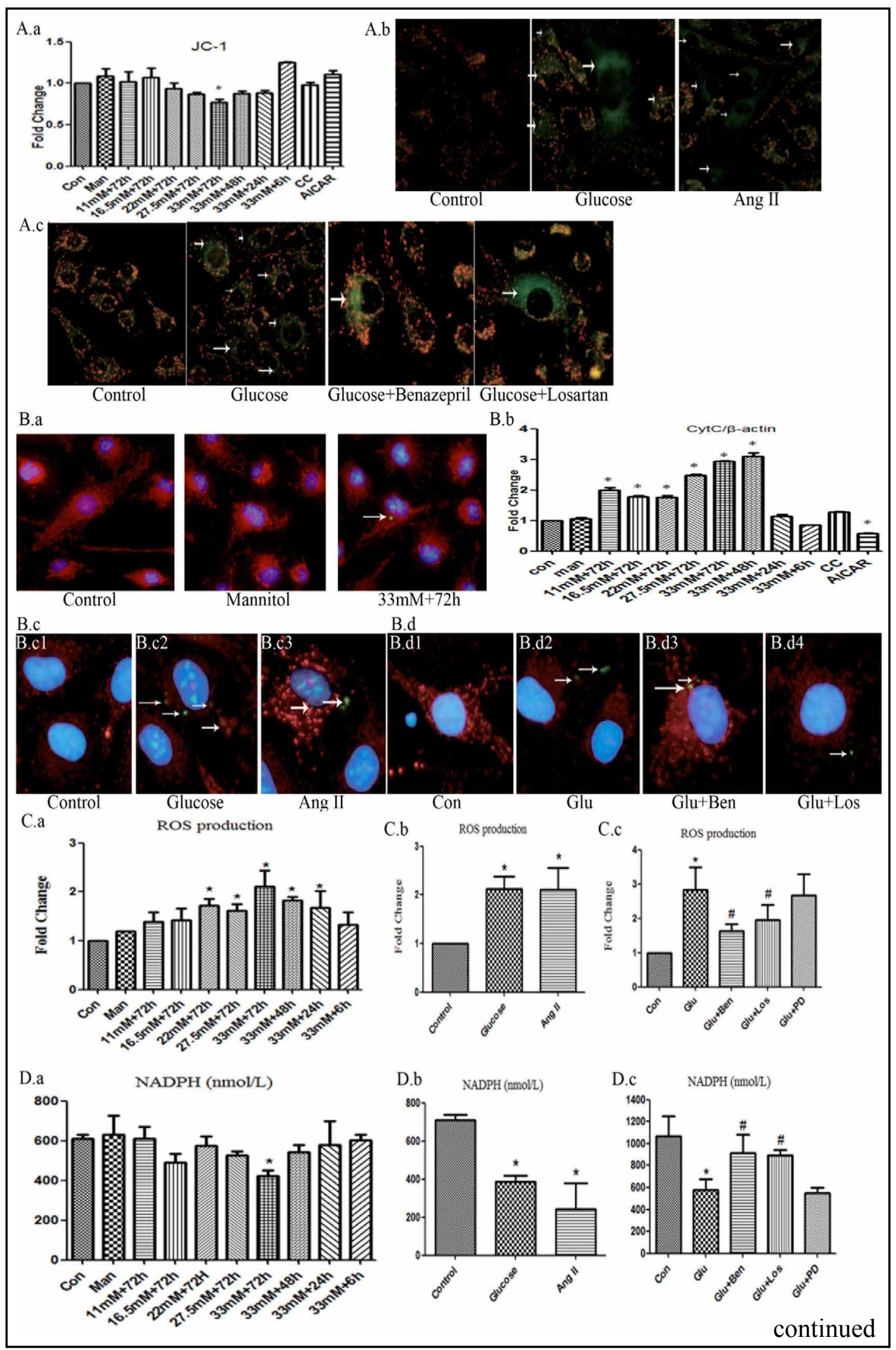

Fig. 2. High-glucose-mediated mitochondrial damage to HUVECs was RAS dependent. Con, control; Glu, glucose; Ben, benazepril; Los, losartan; PD, PD123319. N=3, ${ }^{*} \mathrm{p}<0.05$, compared with control cells; ${ }^{*} \mathrm{p}<0.05$, 


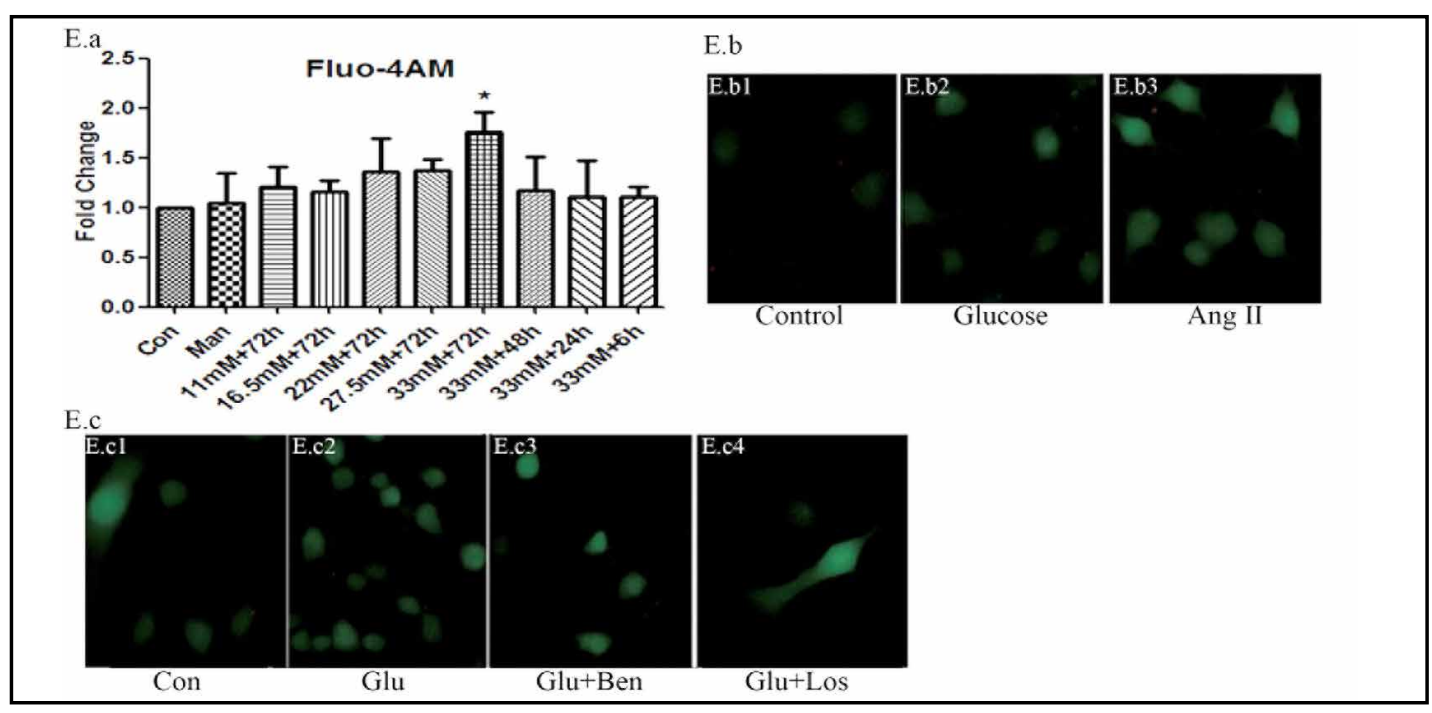

compared with high-glucose-treated cells. A. $\Delta \Psi$ m was detected using JC-1 staining. (a). High-glucose-mediated $\Delta \Psi \mathrm{m}$ loss; (b). Ang mimicked $\Delta \Psi \mathrm{m}$ loss; (c). Benazepril and losartan each ameliorated $\Delta \Psi \mathrm{m}$ loss. Red, normal $\Delta \Psi$ m; green, $\Delta \Psi \mathrm{m}$ loss. B. Representative images showing the distribution of cytC. (a,b). High-glucose-mediated redistribution of cytC and increased expression of total cytC; (c). Ang mimicked cytC decoupling from mitochondria; (d). Benazepril and losartan each ameliorated cytC distribution. Red, mitochondria; green, cytC; blue, nucleus. C. High-glucose-induced increased ROS production (a), which could be mimicked by Ang (b) or ameliorated by benazepril or losartan (c). D. High-glucose reduced NADPH expression (a), which could be mimicked by Ang (b) or increased by benazepril or losartan (c). E. Changes in cytoplasmic $\mathrm{Ca}^{2+}$. (a). High glucose increased cytoplasmic $\mathrm{Ca}^{2+}$ levels. (b). Ang mimicked the increase in cytoplasmic $\mathrm{Ca}^{2+}$. (c). Benazepril and losartan each decreased cytoplasmic $\mathrm{Ca}^{2+}$ levels. Green, Fluo-4AM-positive cells.

substantial increase in total cytC (Fig. 2B). Additionally, $33 \mathrm{mM}$ glucose induced an increase in ROS production and a decrease in NADPH expression. In addition, Fluo-4AM-positive cells increased in number (Fig. 2C-2E).

Then we manipulated Ang to activate the RAS (Fig. 1C). Moreover, ACEI (benazepril) or AGTR1 blockers (losartan) were used to suppress RAS (Fig. 1B). HUVECs exposed to Ang showed similar responses to high glucose, including loss of $\Delta \Psi \mathrm{m}$, redistribution of cytC, increased cytoplasmic $\mathrm{Ca}^{2+}$, increase in ROS production and decrease in NADPH expression, whereas the presence of benazepril or losartan reduced the negative effects (Figs. 2A-2E,3D). Interestingly, the blockage of AGTR2 with PD123319 did not show any beneficial effect (data not shown).

\section{RAS-mitochondria participate in regulating high-glucose-induced cellular dysfunction in HUVEC}

Autophagy, senescence and apoptosis are important responses to mitochondrial damage [6], therefore, increased levels of autophagic biomarkers, including LC3, beclin1 and p62 protein, were firstly investigated (Fig. 3A). Additionally, we noted that high glucose induced LC3 aggregation to one side of the nucleus, with the aggregate shaped as a cap/tube (Fig. 3A). Additionally, cells incubated with high glucose showed increased phosphorylation of AMPK and ULK proteins and expression of PINK1 protein (Fig. 3B), which are the possible underlying promoters of autophagy $[4,6]$. To further reveal the role of the RAS, we observed the effects of Ang, benazepril or losartan on HUVEC autophagy following highglucose treatment. Similar to high glucose, Ang induced a significant increase in the number of autophagic vacuoles per cell and the expression of autophagic biomarkers. Moreover, benazepril and losartan ameliorated the high-glucose-mediated autophagic response (Figs. 3C,3D). 


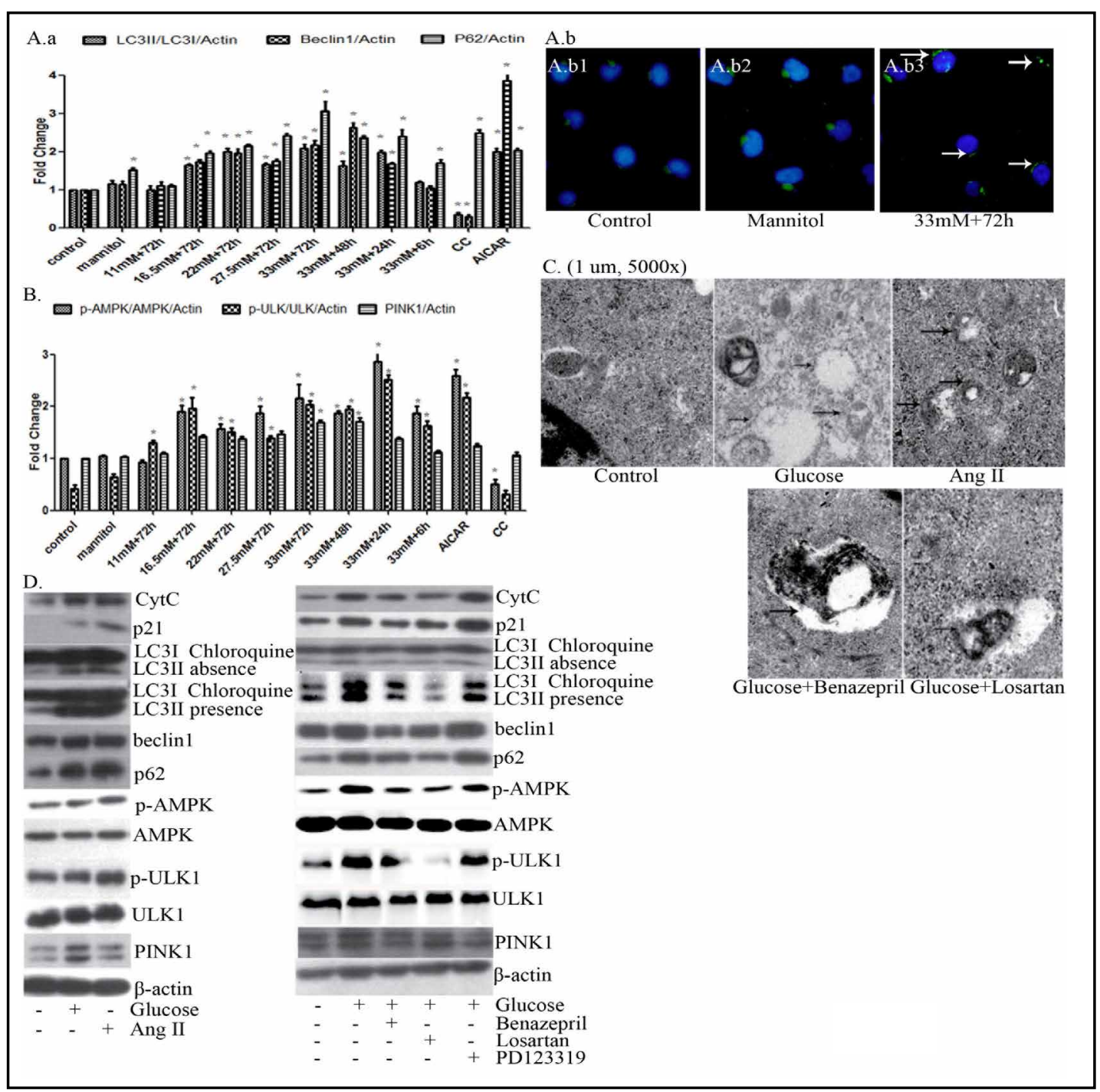

Fig. 3. High-glucose-mediated autophagy to HUVECs was RAS dependent. $N=3,{ }^{*} p<0.05$, compared with control cells. A. (a). High glucose increased protein expression of LC3, beclin1 and p62; (b). High glucose induced LC3 aggregation to one side of nuclei and was shaped as a cap/tube. B. High glucose increased protein expression of autophagic regulators including p-AMPK/AMPK, p-ULK1/ULK1 and PINK1. C. Representative images showing autophagic vacuoles. The double membrane structures of autophagic vacuoles were increased in high-glucose- or Ang-incubated cells and co-administration of benazepril or losartan reduced the number of autophagic vacuoles. D. The protein levels of total cytC, p21, LC3, beclin1, p62, p-AMPK/AMPK, $\mathrm{p}$-ULK/ULK and PINK1. To determine LC3 protein levels, the absence and presence of chloroquine at $10 \mu \mathrm{M}$ for $72 \mathrm{~h}$ was used to reflect the actual intensity of autophagy.

Treatment with glucose led to an increase in SA- $\beta$-gal-positive cells and senescenceassociated heterochromatic foci (SAHF) formation. Meanwhile, high glucose increased the percentage of cells in the G0/G1 and G2/M phases and p21 protein expression (Figs. 4A-4D). Because the regulation of RAS influenced autophagy, we hoped that RAS also participated in senescence. As expected, Ang increased the SA- $\beta$-gal-positive cells, the percentage of cells in G0/G1 or G2/M phases and p21 protein expression, whereas the presence of benazepril or losartan inhibited high-glucose-induced HUVEC senescence (Figs. 3D,4E,4F).

A different result was found with senescence or autophagy; however, as high glucose did not result in apoptosis when labeled with annexin- $V$ (data not shown). We then treated 


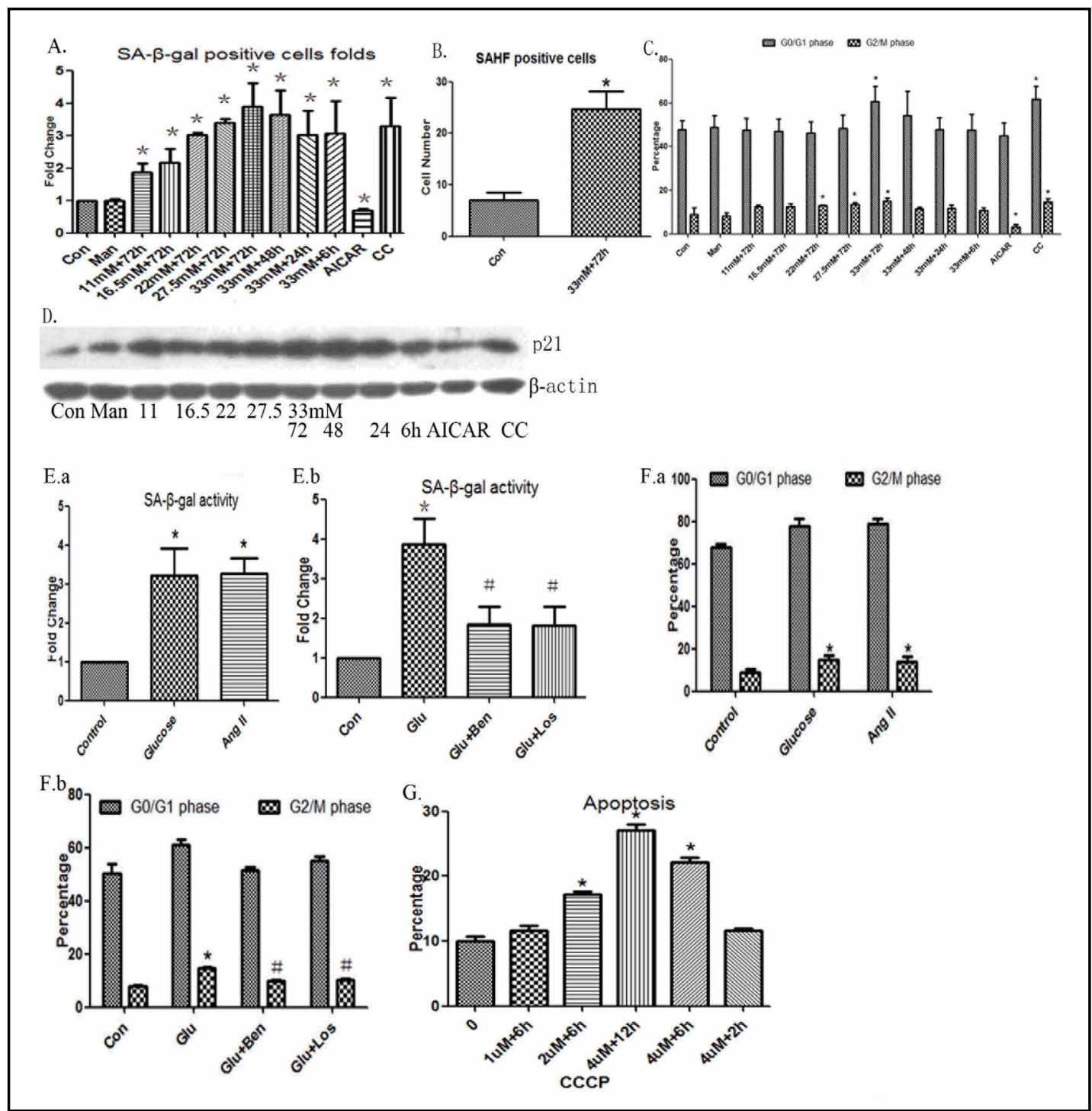

Fig. 4. High-glucose-mediated senescence to HUVECs was RAS dependent. $N=3,{ }^{*} p<0.05$, compared with control cells; ${ }^{\#} \mathrm{p}<0.05$, compared with high-glucose-treated cells. A. High glucose increased the number of SA- $\beta$-gal-positive cells. B. High glucose increased the number of SAHF-positive cells. C. High glucose increased the percentage of G2/M-phase HUVECs. Interestingly, the increase in glucose (33 mM for $72 \mathrm{~h}$ ) paralleled the percentage of G0/G1-phase cells. D. High glucose increased the expression of p21 protein. E. High glucose and Ang increased the number of SA- $\beta$-gal-positive cells (a), which was reversed by co-treatment with benazepril or losartan (b). F. Cell cycle analysis was performed. (a). High glucose and Ang inhibited cell cycle progression in HUVECs; (b). Benazepril and losartan each decreased the fraction of cells in G2/M phase. G. The time- and dose-dependent apoptosis caused by CCCP in HUVECs is shown.

the cells with carbonyl cyanide 3-chlorophenylhydrazone (CCCP) to open the mitochondrial permeability transition pore (mPTP). We noted that CCCP exerted a direct pro-damage action on HUVEC survival, including the loss of $\Delta \Psi \mathrm{m}$, increased expression of p21 protein and autophagic biomarkers, redistribution of cytC (data not shown). But the percentage of cells undergoing apoptosis only increased when the cells were treated with $4 \mu \mathrm{M} \mathrm{CCCP}$ for $12 \mathrm{~h}$ (Fig. 4G). Additionally, the negative effects were not modulated by benazepril or losartan (data not shown). These data indicated that RAS induced HUVEC damage via the mitochondria. 


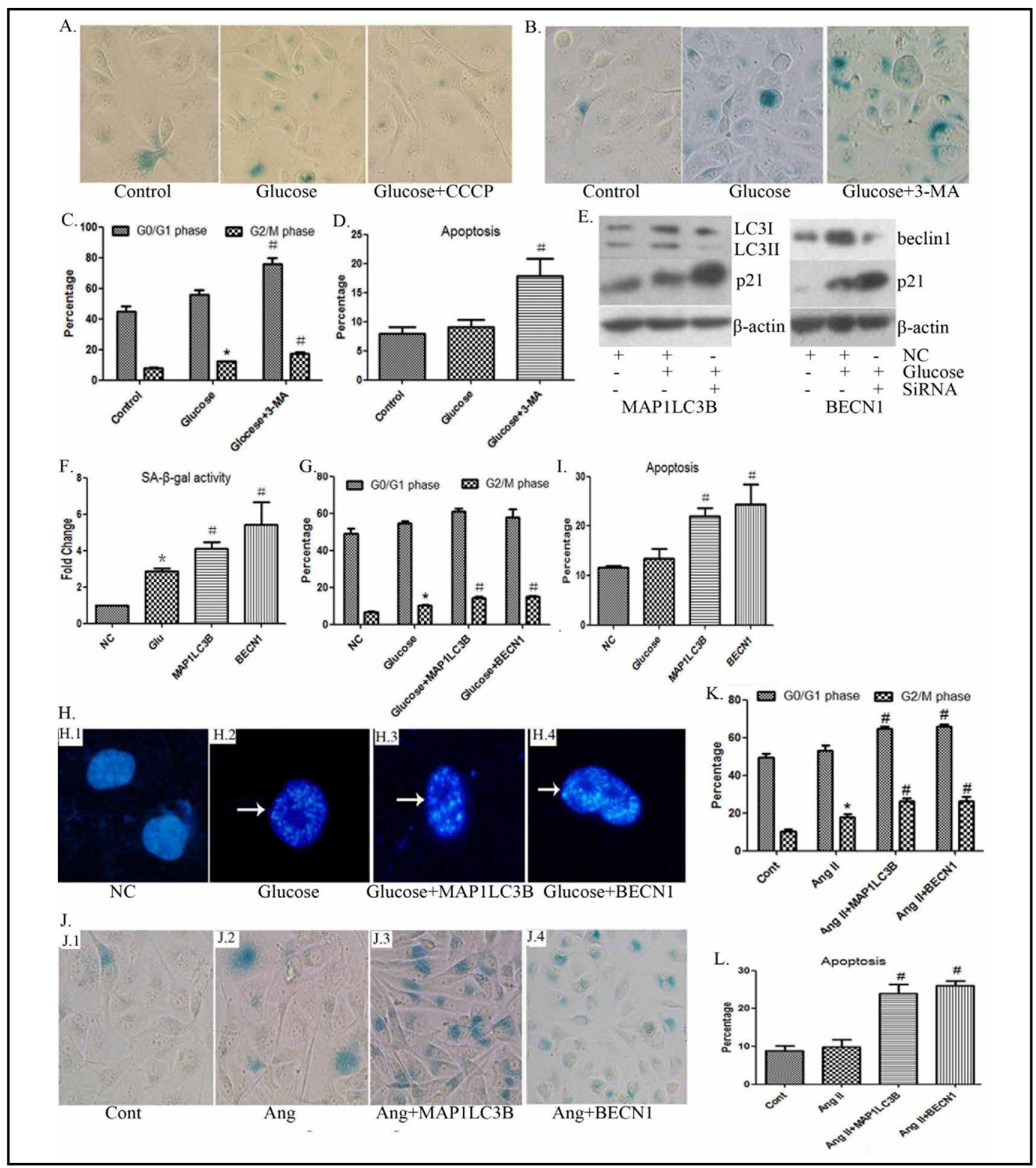

Fig. 5. A deficiency in autophagy enhanced high-glucose- or Ang-mediated damage responses. $N=3,{ }^{*} \mathrm{p}<0.05$ compared with normal cells; \#p<0.05, compared with high-glucose- or Ang-treated cells. A. CCCP $(1 \mu \mathrm{M}$ for $12 \mathrm{~h}$ ) was used to induce abundant autophagy. CCCP inhibited high-glucose-induced senescence. Blue, SA- $\beta$-gal-positive cells. B. Cells were administered with 3-MA at $5 \mathrm{mM}$ for $24 \mathrm{~h}$ to impair autophagy. 3-MA enhanced high-glucose-mediated HUVEC senescence. Blue, SA- $\beta$-gal-positive cells. C. 3-MA enhanced highglucose-mediated cell cycle arrest. D. 3-MA induced apoptosis in HUVECs. Apoptosis analyses were performed using FACS. E. At $24 \mathrm{~h}$ after cells were transfected with MAP1LC3B siRNA, BECN1 siRNA or relevant scrambled siRNAs, cells were cultured in the presence or absence of glucose (33 mM) for an additional 72 h. Cells lysates were subjected to immunoblotting to determine protein levels of p21, LC3 and beclin1. F. SiRNAs enhanced high-glucose-mediated HUVEC senescence. G. Co-treatment with glucose and siRNAs induced an increase in the percentage of G2/M-phase cells. H. High glucose and siRNAs induced typical SAHF structures. Blue, DAPI-labeled nucleus. I. SiRNAs induced an increase in the percentage of apoptotic cells. J. MAP1LC3B SiRNA and BECN1 siRNA significantly increased the number of SA- $\beta$-gal-positive cells. Blue, SA- $\beta$-gal-positive cells. K. Co-treatment with Ang and siRNAs induced an increase in the percentage of G0/ G1 or G2/M-phase cells. L. Co-treatment with Ang and siRNAs increased the percentage of apoptotic cells. 


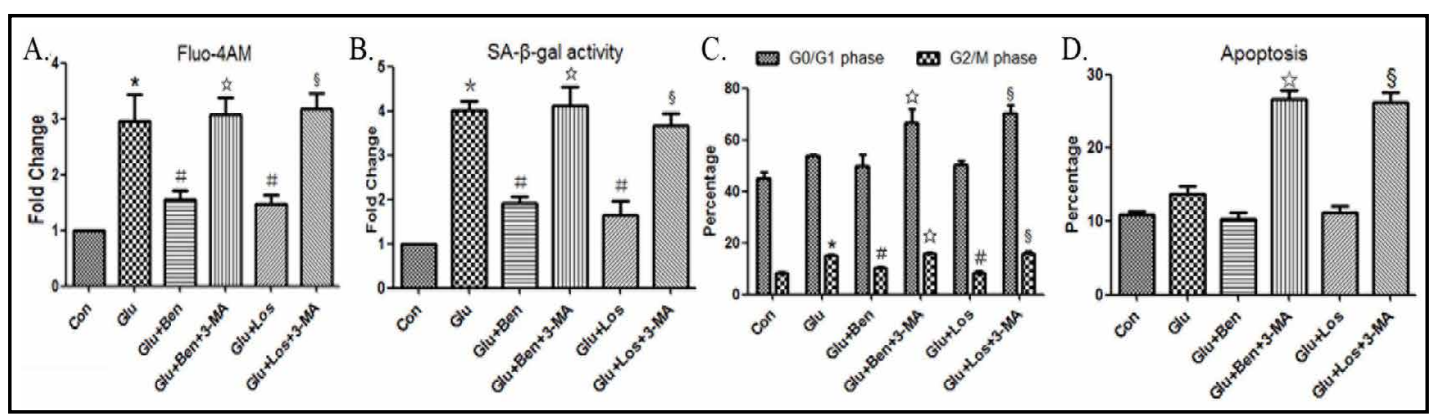

Fig. 6. 3-MA abolished benazepril- or losartan-mediated beneficial effects in high-glucose-treated HUVECs. $\mathrm{N}=3$; ${ }^{*} \mathrm{p}<0.05$, compared with control cells; \# $<<0.05$, compared with high-glucose-treated cells; $i \mathrm{p}<0.05$, compared with cells co-incubated in high glucose with benazepril; $\S p<0.05$, compared with cells co-incubated in high glucose with losartan. A. 3-MA abolished the benazepril or losartan-mediated reduction in cytoplasmic $\mathrm{Ca}^{2+}$ levels. B. When cells were treated with 3-MA, the benazepril- or losartan-induced delay in senescence disappeared. C. We observed an increase in the percentage of G0/G1-phase or G2/M-phase cells in the presence of 3-MA. D. 3-MA induced an increase in the percentage of apoptotic cells in the presence of benazepril or losartan.

High-glucose- or Ang-induced autophagy in HUVECs is associated with senescence and apoptosis

Autophagy biogenesis initiates from the activation of the ULK1 complex through AMPK phosphorylation and the recruitment of the beclin1 complex and elongates under the LC3-phosphatidylethanolamine (PE) complex. Damaged-mitochondria can also activate autophagy through the stabilization of PINK1 and recruitment of PARK2 [18]. First, we used a low dose of CCCP to induce autophagy. As shown in Fig. 5A, the senescence response was reduced. Then, 5-aminoimidazole-4-carboxyamide ribonucleoside (AICAR, an AMPK agonist) and Compound C (CC, an AMPK inhibitor) were used to regulate AMPK activity. AICAR induced AMPK phosphorylation and high-expression of autophagy biomarkers (Figs. 3A,3B); as a result, HUVECs senescence appeared to be reduced. In contrast, the incubation of HUVECs with CC induced the opposite results (Figs. 4A,4C,4D). Moreover, neither AMPK nor $\mathrm{CC}$ affected mitochondrial function or apoptosis (Figs. 2A-2D). Furthermore, we used 3-MA to block autophagosome formation. SA- $\beta$-gal-positive cells and cell cycle arrest in response to high glucose were significantly increased in 3-MA-treated-HUVECs (Figs. 5B,5C). We then generated autophagy-deficient cells by inhibiting the expression of MAP1LC3 and beclin1 (Fig. 5E). Similarly, a robust increase in SA- $\beta$-gal-positive cells, cell cycle arrest, p21 protein expression and typical SAHF formation (Figs. 5E-5H) were observed in these cells. Because autophagy limited the senescence response, we determined that it also regulated apoptosis. As shown in Figs. 5D,5I, autophagy-deficient HUVECs treated with high glucose showed an increase in annexin- $\mathrm{V}$ binding.

Additionally, MAP1LC3B siRNA or BECN1 siRNA was also administrated to Ang-treated HUVECs and caused a robust apoptosis- or senescent-burden upon Ang-induced stress (Figs. 5J-5L). Combining these results, we deduced that autophagy limited the senescence and apoptosis responses to high glucose.

\section{3-MA blocked the benazepril- or losartan-mediated beneficial effects of HUVECS}

Next, we compared the beneficial effects of benazepril or losartan in untreated cells versus 3-MA-treated HUVECs. As shown in Fig. 6, the high-glucose-induced senescence responses and Fluo-4AM-staining burden were enhanced in 3-MA-treated cells but not in untreated cells. Benazepril or losartan induced beneficial effects that disappeared when 3-MA was present. Furthermore, benazepril and losartan did not reverse the 3-MA-induced increase in annexin-V-positive cells. These data confirmed that autophagy limits RASmediated HUVEC damage following high-glucose administration. 

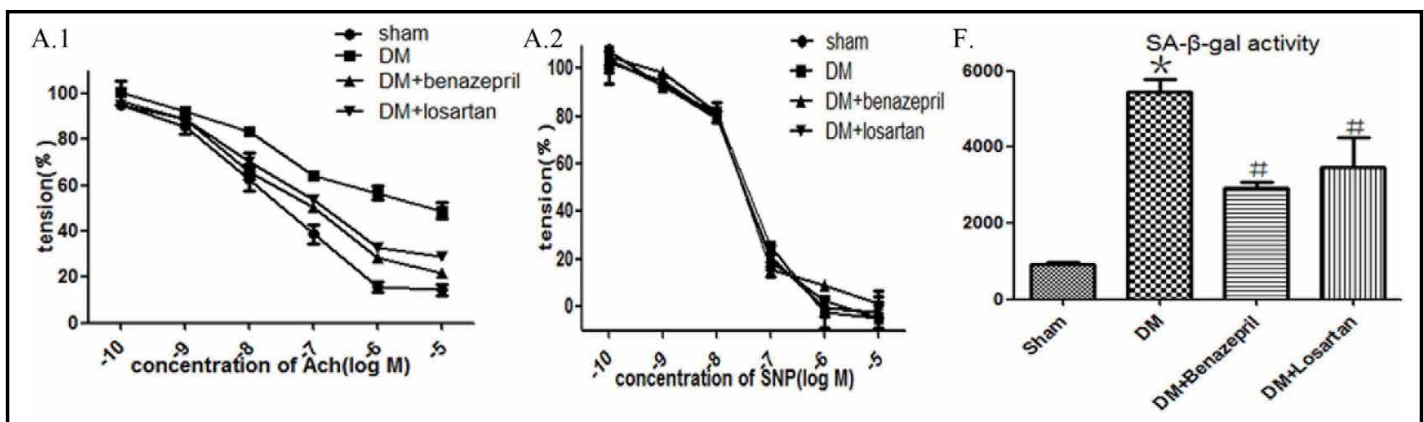

Fig. 7. The accumulation of autophagic, senescent and apoptotic aggregates in diabetic rat aortic endothelium. Sham, normal control rats; DM, untreated diabetic rats; $\mathrm{DM}$ + benazepril, diabetic rats receiving benazepril; DM + losartan, diabetic rats receiving losartan. A. Benazepril or losartan significantly ameliorated diabetes-induced EDR-impairment. Neither diabetes nor RAS inhibitors had any significant effect on EIR. LC3 (B), beclin1 (C) and p62 (D) were absent or faint in blots of endothelial cells from normal control rats whereas an accumulation of LC3, beclin1 and p62positive aggregates were detected in endothelial cells and smooth muscle cells from DM rats. Benazepril and losartan reduced LC3, beclin 1 and p62-positive aggregates in diabetic aortic rings. E. DM induced a significant increase in TUNEL-positive endothelial cell number, and benazepril and losartan reduced TUNEL-positive endothelial cell number. F. Increased activity of SA- $\beta$-gal was observed in diabetic rats and was inhibited following chronic administration of benazepril or losar$\tan . \mathrm{N}=3$ rats.

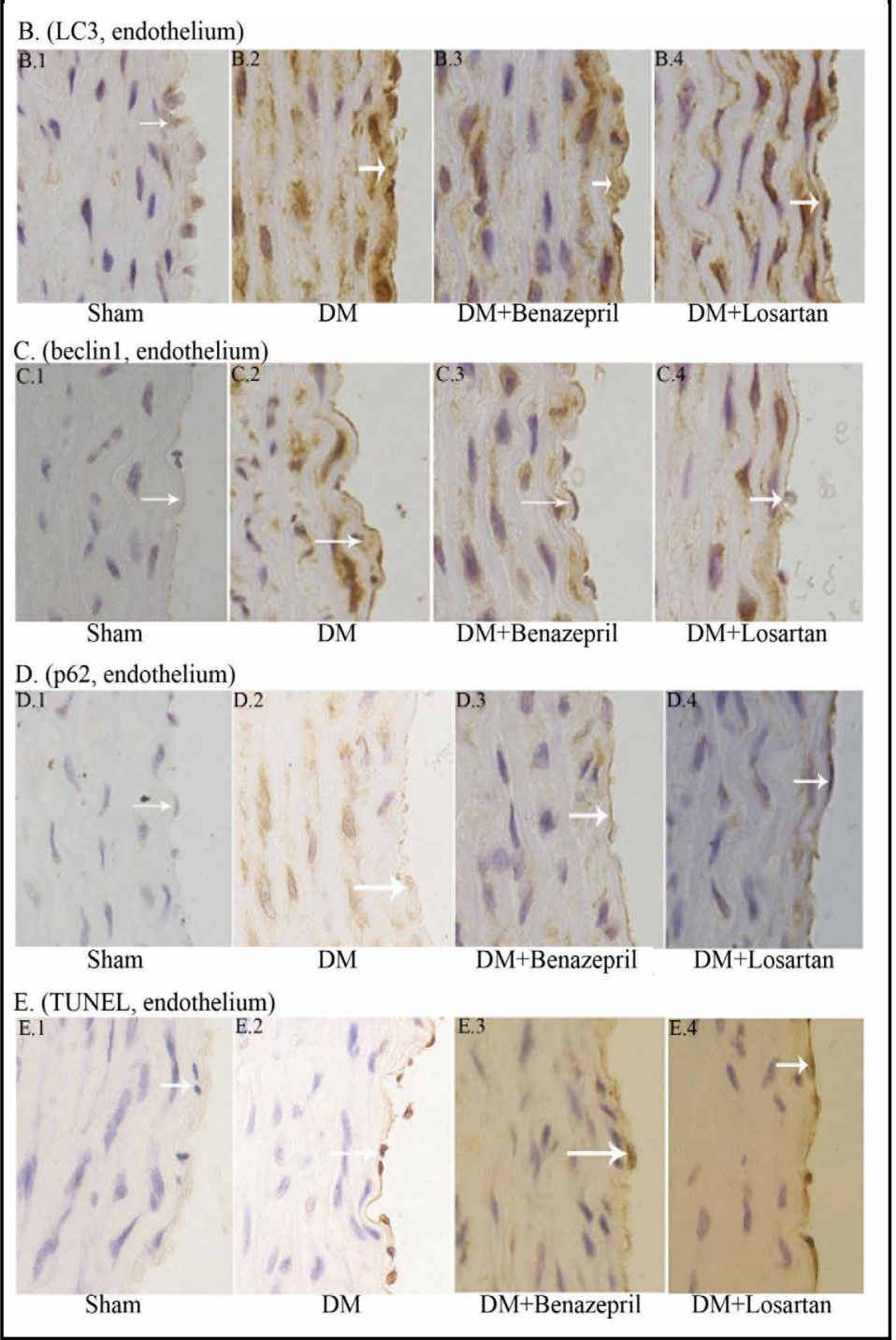

The accumulation of autophagic, senescent and apoptotic aggregates in diabetic rat aortic endothelium

As shown in Fig. 7, acetylcholine (Ach)-induced endothelium-dependent relaxation (EDR) in diabetic rat aortic rings were significantly impaired; the half-maximal effective concentration $\left(\mathrm{EC}_{50}\right)$ of Ach was increased from $85.43 \pm 9.82 \mathrm{nM}$ to $194.77 \pm 8.59 \mathrm{nM}$. Moreover, an accumulation of LC3-, beclin1- and p62-positive aggregates and increased activity of SA$\beta$-gal were observed in endothelial cells and smooth muscle cells. Furthermore, we observed a marked increase in TUNEL-positive endothelial cells. Chronic administration of benazepril 
Fig. 8. Endothelial cells lysates were subjected to immunoblotting to analyze the expression of ACE1, AT1, LC3, beclin1, p62, bax, bcl2 and p21. Consistent with results from cellular and rat tests, increased expression of ACE1, AT1, LC3, beclin1, p62, bax/bcl2 and p21 was observed in tissue from diabetic patients.

or losartan induced beneficial effects in diabetic rats, including an improved EDR; the EC50 decreased to $124.37 \pm 8.27 \mathrm{nM}$ and $110.82 \pm 10.61 \mathrm{nM}$, respectively, and a decrease in the accumulation of autophagic biomarkers, activity of SA- $\beta$-gal and the number of TUNEL-positive cells. Neither diabetes nor RAS inhibitors showed any significant effect on SNP-induced endothelium-independent relaxation (EIR) (Fig. 7A).

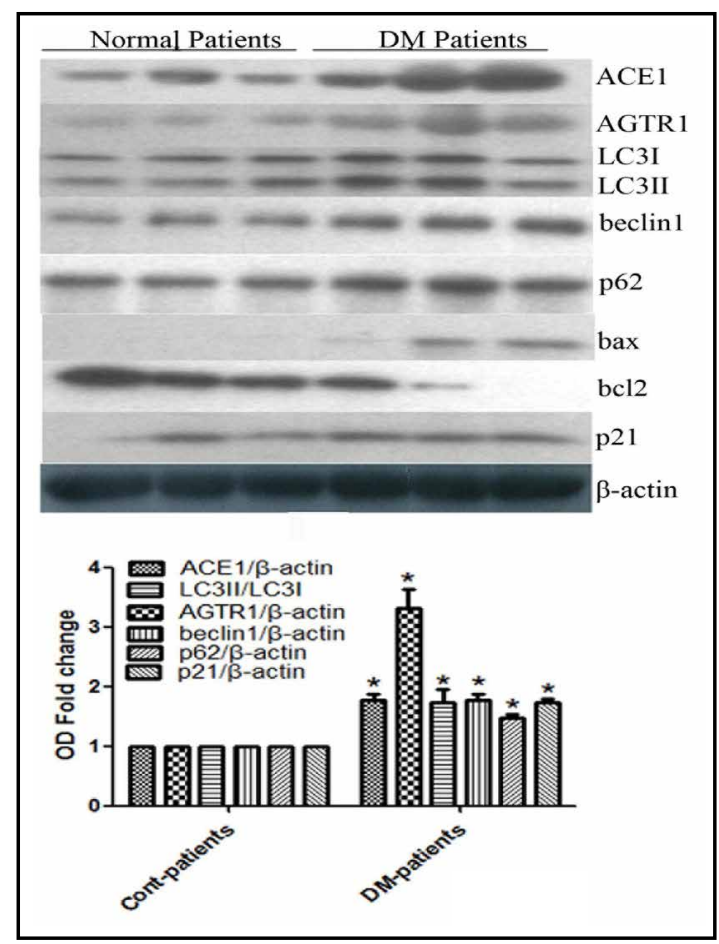

\section{Human Study}

A total of 6 control patients and 7 diabetic patients were enrolled in this study. There were no differences in clinical profiles, such as age, gender and medication use (data not shown). The postprandial glycemia at $2 \mathrm{~h}$ was $8.34 \pm 2.1 \mathrm{mM}$ in the control patients versus $14.16 \pm 3.62 \mathrm{mM}$ in the diabetic patients. After digestion, ratio of the factor-VIII-positive cells to the total collection cells was $77.47 \pm 5.71 \%$. Immunoblotting revealed an increased expression of ACE and AGTR1 proteins in vessels from the diabetic patients. Autophagicor senescent-biomarkers were also detected in the diabetic patients. Furthermore, bax was absent or very faint in blots of the control vessels, whereas its expression was significantly more intense in the vessels from the diabetic patients (Fig. 8). These data suggest that the RAS may be linked to the autophagic, senescent and apoptotic response in diabetic patients.

\section{Discussion}

In this study, we report that high-glucose-induced senescence, apoptosis and autophagic phenotypes in HUVECs, diabetic rats and humans in a RAS-mitochondria-dependent manner. Ang mimics high-glucose-induced HUVEC damage, whereas benazepril or losartan attenuates these negative effects. Direct mitochondrial damage using CCCP abolishes benazepril- or losartan-mediated beneficial effects. Moreover, a deficiency of autophagy using siRNA or pharmacologic therapy accelerates high-glucose- or Ang-induced senescence and apoptosis and even abrogates benazepril- or losartan-mediated beneficial effects. Additionally, activated autophagy using AICAR or a low dose of CCCP reduces senescence. Thus, autophagy protects against senescence and apoptosis via the RAS-mitochondria in high-glucose-induced endothelial cells.

High-glucose is a pluripotent toxic factor and an important contributor to cardiomyopathy, atherosclerosis, hypertension and glomerulosclerosis [5]. The mechanism by which highglucose triggers cell damage and to what degree the different forms of damage are initiated, including autophagy, senescence and apoptosis, remain unclear. RAS has traditionally been thought to be critical for the development of cardiovascular diseases [19]; therefore it is also a candidate for being a glucose target. In the present study, we indicated that high-glucose- 
induced high expression of ACE1 and AGTR1 and regulated RAS activity could influence HUVEC damage, which verified the potential target role of RAS.

At present, it is believed that many RAS-initiated effects are currently accepted to be mediated by mitochondrial-ROS. Exogenous Ang-caused-senescence is inhibited by valsartan or superoxide dismutase [SOD; 20-22]. Moreover, the overexpression of catalase targeted to mitochondrial in mice appears resistant to hypertrophy, mitochondrial damage and heart failure induced by Ang or by the overexpression of Gaq [17]. Additionally, activatedNADPH oxidation increases mitochondrial ROS production in Ang-induced endothelial cells [20]. Addiotionally, Ang has been shown to interact with $\mathrm{Ca}^{2+}$-mediated signaling [17]. In the present study, high glucose or Ang significantly induced a decrease in $\Delta \Psi \mathrm{m}$ decrease, cytC redistribution, a $\mathrm{Ca}^{2+}$ overload, increased ROS production and a decrease in NADPH. Moreover, benazepril or losartan inhibited mitochondrial dysfunction. Additionally, the opening of mPTP abolished benazepril- or losartan-mediated beneficial effects. These data confirmed the causal role of RAS-induced mitochondrial damage in high-glucose-induced HUVECS.

Mitochondria-ROS acts as a central regulator of RAS-mediated cell damage, such as autophagy, senescence and apoptosis. Autophagy is initiated from damaged mitochondria through different manners then completes the degradation of the damaged mitochondria; it is therefore a very important process for mitochondrial quality control. First, damaged mitochondria-induced ROS production and energy depletion stimulates the phosphorylation of AMPK.AMPK phosphorylation is essential to control the activity and proper phosphorylation of ULK1, which forms a stable complex with autophagy-related (Atg) proteins. Autophagy initiates from the recruitment of the ULK1 complex and beclin 1 complex for the isolation of membrane formation [23]. Second, the loss of $\Delta \Psi \mathrm{m}$, which is induced by mitochondrial depolarization, stabilizes PINK1, a mitochondrial outer membrane-associated protein. Aggregation of PINK1 recruits PARK2, an E3 ubiquitin ligase, from the cytoplasm to the damaged mitochondria. PARK2 causes ubiquitination of the outer mitochondrial membrane proteins. The ubiquitinated mitochondria are recruited to the early-stage autophagosome through p62 binding [24]. Although controversial, p62 accumulation is typically interpreted as a symptom of lysosomal degradation and the exact opposite to enhanced autophagy flux [25-27]. The increase in p62 may indicate that autophagy is not sufficient to process the damaged proteins bound to p62. Then, mitophagy receptors BNIP3 and FUNDC1 directly present the damaged mitochondria to autophagosomes through LC3-binding [18]. Moreover, the mitochondrial localization of ERK2 has been reported to initiate autophagy [28]. In our test, Ang mimicked high-glucose-induced autophagy in HUVECs, including the phosphorylation of AMPK-ULK, the expression of PINK1-p62, LC3 and beclin1 and the formation of the autophagosome, whereas benazepril or losartan ameliorated high-glucoseinduced autophagic responses, which were reversed by CCCP. These data prove that the RASmitochondria pathway plays a critical role in high-glucose-induced autophagy in HUVECs. Furthermore, if autophagy failed to digest the damaged mitochondria, mPTP opening would extensively induce serious $\Delta \Psi \mathrm{m}$ loss, abundant ROS production and cytC release and affluent caspase activation. Then, the damaged cells would dye through apoptosis or necrosis [29]. In our present study, although high glucose, Ang or a low dosage of CCCP induced mitochondrial damage, the percentage of apoptotic cells was unchanged, whereas a high dosage of CCCP increased the percentage of apoptotic cells. Additionally, the TUNEL-positive endothelial cells or bax/bcl2 expression increased in diabetic rats or humans, which would be reduced by using benazepril or losartan. Apart from autophagy and apoptosis, mitochondrial-ROS contributes to RAS-induced cell senescence. ROS-induced protein S-nitrosylation seems to be the major contributor, while sulfhydryl-ACEI promotes endothelial cell survival and delays senescence $[15,30]$. The present study provides evidence that high-glucose- or Ang-induced mitochondrial ROS increase causes increases in SA- $\beta$-gal- or SAHF-positive cell numbers, cell cycle arrest and p21 protein expression. Moreover, benazepril or losartan inhibits senescence, which is abolished by using CCCP. These data reveal that the RAS-mitochondriaROS play key roles in high-glucose-induced endothelial senescence. 
Current evidence supports the fact that autophagy appears to mediate resistance to apoptosis, whereas increased apoptotic cells appear if autophagy is blocked. In contrast, beclin1, a major component of the autophagosome, combines with the anti-apoptotic Bcl2 family to impair autophagy in senescent fibroblasts [9-11, 31, 32]. Beclin1 expression decreases in senescent cells and older tissues in parallel with an increase in the expression of the Bcl-2 family and a lower lysosomal uptake of binding substrates [33, 34]. Thus, autophagy inhibition accelerates the development of senescence and apoptosis, although this theory is controversial [10]. The co-regulation of p62 and LC3 causes dual effects on autophagic and senescent responses: decreases in autophagic and senescent programs or increases in autophagic and senescent biomarkers have been reported [35]. This paradox also appears in the inhibition of atg5, atg7 and atg3, which are accompanied by no change in apoptosis [3638]. In this study, we confirmed that autophagy limited senescent and apoptotic responses in high-glucose- or Ang-treated HUVECs. Targeting autophagy induced robust SA- $\beta$-gal staining and increased cell cycle arrest, p21 protein expression, SAHF formation and even newly presenting annexin-V-positive cells. Interestingly, TUNEL-positive endothelial cells or increased bax/bcl2 expression were observed in diabetic rats or patients with intact autophagic mechanisms. This discrepancy may be attributed to variability in the time of high glucose administration. Although autophagic-level increases in Ang-treated heart muscle or myocardiocytes [10-15], the autophagic pathway has been shown to play a cytoprotective role in maintaining cardiac function $[39,40]$. In our present study, RAS-induced autophagy seems to mediate a resistance to apoptosis and senescence in high-glucose- or Ang-treated HUVECs, while 3-MA abolishes benazepril- or losartan-reduced senescence and the apoptotic cells increase. These data provide evidence that autophagy limits RAS-mitochondrial-ROSmediated cell damage in high-glucose-induced HUVECs.

In conclusion, high glucose induced increased autophagy, senescence and apoptotic responses via RAS-mediated mitochondrial damage in endothelial cells. A deficiency in autophagy enhanced the senescence response and caused apoptosis, whereas it inhibited the beneficial effects induced by benazepril and losartan. Many questions must be answered regarding the molecular network underlying these responses. However, the possibility of the essential role of autophagy on RAS-mediated cellular damage is very exciting.

\section{Acknowledgements}

Thanks for Norvatis Cop. to provide benazepril kindly. We also thanks for Prof. Ji-Ren Yu to provide clinical samples kindly. Moreover, we thanks for Prof. Fei Zheng to help us to carry out partially western-blotting test. This work was supported by the China National Natural Science Fundation, Nos.: 30470715, 30870939 and 81170242.

\section{Disclosure Statement}

None conflict of interest.

\section{References}

1 Meenal P, Ayako K: Mitochondrial function in vascular endothelial cell in diabetes. J Smooth Muscle Res 2012;48:1-26.

-2 Dejana E, Tournier LE, Weinstein BM: The control of vascular integrity by endothelial cell junctions: molecular basis and pathological implications. Dev Cell 2009;169:209-221.

-3 Yokoi T, Fukuo K, Yasuda O, Hotta M, Miyazaki J, Takemura Y, Kawamoto H, Ichijo H, Ogihara T: Apoptosis signal-regulating kinase1 mediates cellular senescence induced by high glucose in endothelial cells. Diabetes 2006;55:1660-1665. 
4 Contreras L, Drago I, Zampese E, Pozzan T: Mitochondia: the calcium connection. Biochim Biophys Acta 2010;1797:607-618.

-5 Bhatt MP, Lim YC, Kim YM, Ha KS: C-peptide activates AMPK $\alpha$ and prevents ROS-mediated mitochondrial fission and endothelial apoptosis in diabetes mellitus. Diabetes 2013;66:3851-3862.

6 Klionsky DJ, Abdalla FC, Abeliovich H, Abraham RT, Acevedo-Arozena A, Adeli K, et al: Guidelines for the use and interpretation of assays for monitoring autophagy. Autophagy 2012;8:445-544.

7 Wang X, Dai Y, Ding Z, Khaidakov M, Mercanti F, Mehta JL: Regulation of autophagy and apoptosis in response to angiotensin II in HL-1 cardiomyocytes. Biochem Biophys Res Commun 2013;440:696-700.

8 Xiong S, Salazar G, Patrushev N, Ma M, Forouzandeh F, Hilenski L, Alexander RW: Peroxisome proliferatoractivated receptor $\gamma$ coactivator- $1 \alpha$ is a central negative regulator of vascular senescence. Arterioscler Thromb Vasc Biol 2013;33:988-998.

9 Gebel S, Lichtner RB, Frushour B, Schlage WK, Hoang V, Talikka M, Hengstermann A, Mathis C, Veljkovic E, Peck M, Peitsch MC, Deehan R, Hoeng J, Westra JW: Construction of a computable network model for DNA damage, autophagy, cell death, and senescence. Bioinformatics and Biology Insights 2013;7:97-117.

10 Salminen A, Kaarniranta K, Kauppinen A: Beclin 1 interactome controls the crosstalk between apoptosis, autophagy and inflammasome activation: Impact on the aging process. Ageing Res Rev 2013;2013,12:520534 .

11 Salminen A, Kauppinen A, Kaarniranta K: Emerging role of NF- $\kappa B$ signaling in the induction of senescenceassociated secretory phenotype (SASP). Cellular Signal 2012;24:835-845.

12 Porrello ER, D'Amore A, Curl CL, Allen AM, Harrap SB, Thomas WG, Delbridge LM: Angiotensin II Type 2 Receptor Antagonizes Angiotensin II Type 1 Receptor-Mediated Cardiomyocyte Autophagy. Hypertension 2009;53:1032-1040.

13 Ichihara S, Senbonmatsu T, Price EJ, Ichiki T, Gaffney FA, Inagami T: Angiotensin II type 2 receptor is essential for left ventricular hypertrophy and cardiac fibrosis in chronic angiotensin II-induced hypertension. Circulation 2001;104:346-351.

14 Senbonmatsu T, Ichihara S, Price EJ, Gaffney FA, Inagami T: Evidence for angiotensin II type 2 receptormediated cardiac myocyte enlargement during in vivo pressure overload. J Clin Invest 2000;106:R25-R29.

-15 Sandra D, Erika T, Marina Z, Lucia M: Sulfhydryl angiotensin-converting enzyme inhibitor promotes endothelial cell survival through nitric-oxide synthase, fibroblast growth factor-2, and telomerase crosstalk. J Pharmacol Exp Ther 2010;332:776-784.

16 Enzo RP, Lea MDD: Cardiomyocyte autophagy is regulated by angiotensin II type 1 and type 2 receptors. Autophagy 2009;5:1215-1216.

17 Dai DF, Johnson SC, Villarin JJ, Chin MT, Nieves-Cintrón M, Chen T, Marcinek DJ, Dorn GW 2nd, Kang YJ, Prolla TA, Santana LF, Rabinovitch PS: Mitochondrial oxidative stress mediates angiotensin II-induced cardiac hypertrophy and Galphaq overexpression-induced heart failure. Circ Res 2011;108:837-846.

18 Feng D, Liu L, Zhu Y, Chen Q: Molecular signaling toward mitophagy and its physiological significance. Exp Cell Res 2013;319:1697-1705.

-19 De Giusti VC, Caldiz CI, Ennis IL, Pérez NG, Cingolani HE, Aiello EA: Mitochondrial reactive oxygen species (ROS) as signaling molecules of intracellular pathways triggered by the cardiac renin-angiotensin IIaldosterone system (RAAS). Front Physiol 2013;4:126.

20 Imanishi T, Tsujioka H, Akasaka T: Endothelial progenitor cells dysfunction and senescence: contribution to oxidative stress. Curr Cardiol Rev 2008;4:275-286.

-21 Sowers JR: Hypertension, angiotensin II, and oxidative stress. N Engl J Med 2002;346:1999-2001.

22 Doughan AK, Harrison DG, Dikalov SI: Molecular mechanisms of angiotensin II-mediated mitochondrial dysfunction: linking mitochondrial oxidative damage and vascular endothelial dysfunction. Circ Res 2008; 102:488-496.

23 Egan DF, Shackelford DB, Mihaylova MM, Gelino S, Kohnz RA, Mair W, Vasquez DS, Joshi A, Gwinn DM, Taylor R, Asara JM, Fitzpatrick J, Dillin A, Viollet B, Kundu M, Hansen M, Shaw RJ: Phosphorylation of ULK1 (hATG1) by AMP-activated protein kinase connects energy sensing to mitophagy. Science 2011;331:456461.

24 Bellot GL, Liu D, Pervaiz S: ROS, autophagy, mitochondria and cancer: Ras, the hidden master? Mitochondrion 2013;13:155-162.

25 Zhou C, Huang Y, Shao Y, May J, Prou D, Perier C, Dauer W, Schon EA, Przedborski S : The kinase domain of mitochondrial PINK1 faces the cytoplasm. Proc Natl Acad Sci USA 2008;105: 12022-12027. 
26 Heeman B, Van den Haute C, Aelvoet SA, Valsecchi F, Rodenburg RJ, Reumers V, Debyser Z, Callewaert G, Koopman WJ, Willems PH, Baekelandt V: Depletion of PINK1 affects mitochondrial metabolism, calcium homeostasis and energy maintenance. J Cell Sci 2011;124:1115-1125.

27 Patschan S, Chen J, Gealekman O, Krupincza K, Wang M, Shu L, Shayman JA, Goligorsky MS: Mapping mechanisms and charting the time course of premature cell senescence and apoptosis: lysosomal dysfunction and ganglioside accumulation in endothelial cells. Am J Physiol Renal Physiol 2008;294:F100109.

28 Dagda RK, Zhu J, Kulich SM, Chu CT: Mitochondrially localized ERK2 regulates mitophagy and autophagic cell stress: implications for Parkinson's disease. Autophagy 2008;4:770-782.

29 Chen H, McCaffery JM, Chan DC: Mitochondrial fusion protects against neurodegeneration in the cerebellum. Cell 2007;130:548-562.

30 Vamos Z, Cseplo P, Ivic I, Matics R, Hamar J, Koller A: Age determines the magnitudes of angiotensin IIinduced contractions, $\mathrm{mRNA}$, and protein expression of angiotensin type 1 receptors in rat carotid arteries. J Gerontol A Biol Sci Med Sci doi: 10.1093/gerona/glt128.

-31 He C, Levine B: The Beclin 1 interactome. Curr Opin Cell Biol 2010:22:140-149.

32 Kang R, Zeh HJ, Lotze MT, Tang D: The Beclin1 network regulates autophagy and apoptosis. Cell Death Differ 2011;18:571-580.

33 Cuervo AM: Autophagy and aging: keeping that old broom working. Trends Genet 2008;24:604-612.

34 Cho YY, Kim DJ, Lee HS, Jeong CH, Cho EJ, Kim MO, Byun S, Lee KY, Yao K, Carper A, Langfald A, Bode AM, Dong Z: Autophagy and cellular senescence mediated by sox2 suppress malignancy of cancer cells. PLoS One 2013;8:e57172.

35 Motoko S, Masami M, Yasunori S, Yasuni N: A possible involvement of p62/sequestosome-1 in the process of biliary epithelial autophagy and senescence in primary biliary cirrhosis. Liver Int 2012;32:487-499.

-36 Leidal AW, Lee PWK, McCormick C: Viral subversion of autophagy impairs oncogene-induced senescence. Autophagy 2012;8:1138-1140.

-37 Leidal AW, Cyr DP, Hill RJ, Lee PWK, McCormick C: Subversion of autophagy by Kaposi's sarcoma-associated herpesvirus impairs oncogene-induced senescence. Cell Host Microbe 2012;11:167-180.

- 38 Drullion C, Trégoat C, Lagarde V, Tan S, Gioia R, Priault M, Djavaheri-Mergny M, Brisson A, Auberger P, Mahon FX, Pasquet JM: Apoptosis and autophagy have opposite roles on imatinib-induced K562 leukemia cell senescence. Cell Death Dis 2012;3:e373.

-39 Xu X, Pacheco BD, Leng L, Bucala R, Ren J: Macrophage migration inhibitory factor plays a permissive role in the maintenance of cardiac contractile function under starvation through regulation of autophagy. Cardiovasc Res 2013;99:412-421.

40 Bastien NR, Ciuffo GM, Saavedra JM, Lambert C: Angiotensin II receptor expression in the conduction system and arterial duct of neonatal and adult rat hearts. Regul Pept 1996;63:9-16. 